\title{
Using a rapid environmental scan methodology to map country-level global health research expertise in Canada
}

Ranjana Nagi ${ }^{1}$, Susan Rogers Van Katwyk ${ }^{1,2}$ and Steven J. Hoffman ${ }^{1,2,3,4^{*}}$ (D)

\begin{abstract}
Background: Many countries are currently rethinking their global health research funding priorities. When resources are limited, it is important to understand and use information about existing research strengths to inform research strategies and investments and to drive impact. This study describes a method to rapidly assess a country's global health research expertise and applies this method in the Canadian context.

Methods: We developed a three-pronged rapid environmental scan to evaluate Canadian global health research expertise that focused on research funding inputs, research activities and research outputs. We assessed research funding inputs from Canada's national health research funding agency and identified the 30 Canadian universities that received the most global health research funding. We systematically searched university websites and secondary databases to identify research activities, including research centres, research chairs and research training programmes. To evaluate research outputs, we searched PubMed to identify global health research publications by Canadian university-affiliated researchers. We used these three perspectives to develop a more nuanced understanding of Canadian strengths in global health research from different perspectives.
\end{abstract}

Results: Canada's main global health research funder, the Canadian Institutes of Health Research, invested a total of \$314 M from 2000 to 2016 on global health research grants. This investment has contributed to Canada's wealth of global health research expertise, including 12 training programmes, 27 Canada Research Chairs, 6 research centres and 30 WHO Collaborating Centres across 27 universities. Research activities were concentrated in Canada's biggest cities and most commonly focused on health equity and globalisation issues. Canadian-affiliated researchers have contributed to a research output of 822 unique publications on PubMed. There is an opportunity to build global health expertise in regions not already concentrated with research activity, focusing on transnational risks and neglected conditions research.

(Continued on next page)

\footnotetext{
* Correspondence: steven.hoffman@globalstrategylab.org

${ }^{1}$ Global Strategy Lab, Dahdaleh Institute for Global Health Research, Faculty of Health, and Osgoode Hall Law School, York University, 4700 Keele Street 2120 Dahdaleh Building, Toronto, Ontario M3J 1P3, Canada

${ }^{2}$ School of Epidemiology and Public Health, Faculty of Medicine, University of Ottawa, Ottawa, Ontario, Canada

Full list of author information is available at the end of the article
}

C C The Author(s). 2020 Open Access This article is licensed under a Creative Commons Attribution 4.0 International License, which permits use, sharing, adaptation, distribution and reproduction in any medium or format, as long as you give appropriate credit to the original author(s) and the source, provide a link to the Creative Commons licence, and indicate if changes were made. The images or other third party material in this article are included in the article's Creative Commons. licence, unless indicated otherwise in a credit line to the material. If material is not included in the article's Creative Commons licence and your intended use is not permitted by statutory regulation or exceeds the permitted use, you will need to obtain permission directly from the copyright holder. To view a copy of this licence, visit http://creativecommons.org/licenses/by/4.0/. The Creative Commons Public Domain Dedication waiver (http://creativecommons.org/publicdomain/zero/1.0/) applies to the data made available in this article, unless otherwise stated in a credit line to the data. 
(Continued from previous page)

Conclusions: Our three-pronged approach allowed us to rapidly identify clear geographic and substantive areas of strength in Canadian global health research, including urban regions and research focused on health equity and globalisation topics. This information can be used to support research policy directives, including to inform a Canadian global health research strategy, and to allow relevant academic institutions and funding organisations to make more strategic decisions regarding their future investments.

Keywords: Global health, Meta-research, Capacity-building, Canada

\section{Background}

The global health research funding landscape is complex, with multiple public and private groups that vary greatly in their financing capacities and research interests. Despite the variation, a large proportion of health research funding comes from funding bodies in high-income countries [1]. As national priorities shift and funders explore new ways of setting research agendas [2], it is important that strategies are informed by knowledge of the relevant jurisdiction's current strengths and future opportunities. Building on strengths is particularly beneficial when aiming to maximise limited research funding [3] and identifying existing research strengths in global health can assist countries in effectively leveraging these domestic assets in their foreign policy and international development efforts [4].

In the Canadian context, research funders are estimated to collectively invest $\mathrm{C} \$ 90$ million per year in global health research activities [5]. Yet, despite this sum, the Canadian funding landscape for global health has been criticised for being uncoordinated and inefficient [5]. Box 1 describes the roles of Canada's main global health funders; however, as a 'middle power', Canada cannot meaningfully invest in all areas of global health research concomitantly. Identifying existing strengths in research expertise may prove difficult, especially for countries with limited resource capacity. Despite the range of literature on programme implementation and evaluation frameworks, to the best of our knowledge, there is no existing framework that can be used to capture research expertise at the national level. We sought to fill this gap by developing a framework that provides a systematic approach to identifying expertise by drawing upon a range of research inputs, activities and outputs. In this manuscript we aimed to (1) summarise and critically evaluate the available evidence on global health research expertise at Canadian universities through research inputs, activities and outputs (Fig. 1), and (2) assess Canada's overall global health research expertise using strengths in select research inputs, activities and outputs [6, 7].

\section{Box 1. Global health funding bodies in Canada}

Global health in Canada is predominantly funded by three agencies:

1. Canadian Institutes of Health Research: a federal funding agency that supports health research across four pillars (biomedical, clinical, health systems services and population health) [8]

2. International Development Research Centre: a Canadian Crown corporation that supports research in developing countries as a part of Canada's foreign affairs and development efforts [8]

3. Grand Challenges Canada: a non-profit receiving financial support primarily from the government through Global Affairs Canada, funds innovators in low- and middle-income countries and Canada $[9,10]$

\section{Methods}

We developed a three-pronged analytic framework that includes research inputs, activities and outputs. For this assessment, research 'inputs' were global health research funding from Canadian Institutes of Health Research (CIHR) - the Canadian funder with the largest focus on domestic global health research capacity. Research 'activities' included research training programmes in academic institutions, programmes aimed at recruiting top research talent in the country (e.g. Canada Research Chairs (CRC) Program), research centres at these institutions and formal research partnerships with multilateral health organisations (e.g. WHO Collaborating Centres). To varying degrees, these activities contribute to research 'outputs' such as published literature [11-13].

\section{Study definitions, sample and qualitative coding}

We defined global health research as research focusing on health, health systems, health inequities and health policy challenges facing populations living in conditions of vulnerability in both low- and middle-income countries (LMICs) and high-income countries [7]. We identified research activities by systematically searching for research training programmes, research centres and research chairs focused on global health. We mapped research outputs by identifying highly cited authors, their affiliated universities and their 
areas of research from PubMed. For research inputs, we systematically analysed an administrative dataset of global health research funding in Canada from 2000 to 2016, specifically focusing our analysis on the 30 research institutions in Canada that received the most global health research funding from CIHR over this 15-year period [7].

Database and web searches were conducted in March 2018. When applicable, we coded activities and outputs using a previously developed system of 13 research areas and four research themes (Box 2), which are consistent with the CIHR's mandates, research pillars and institutes [7, 14]. When activities and outputs pertained to multiple foci, they were coded using the most-fitting primary focus. Activities and outputs were coded as 'other' when there was inadequate information.

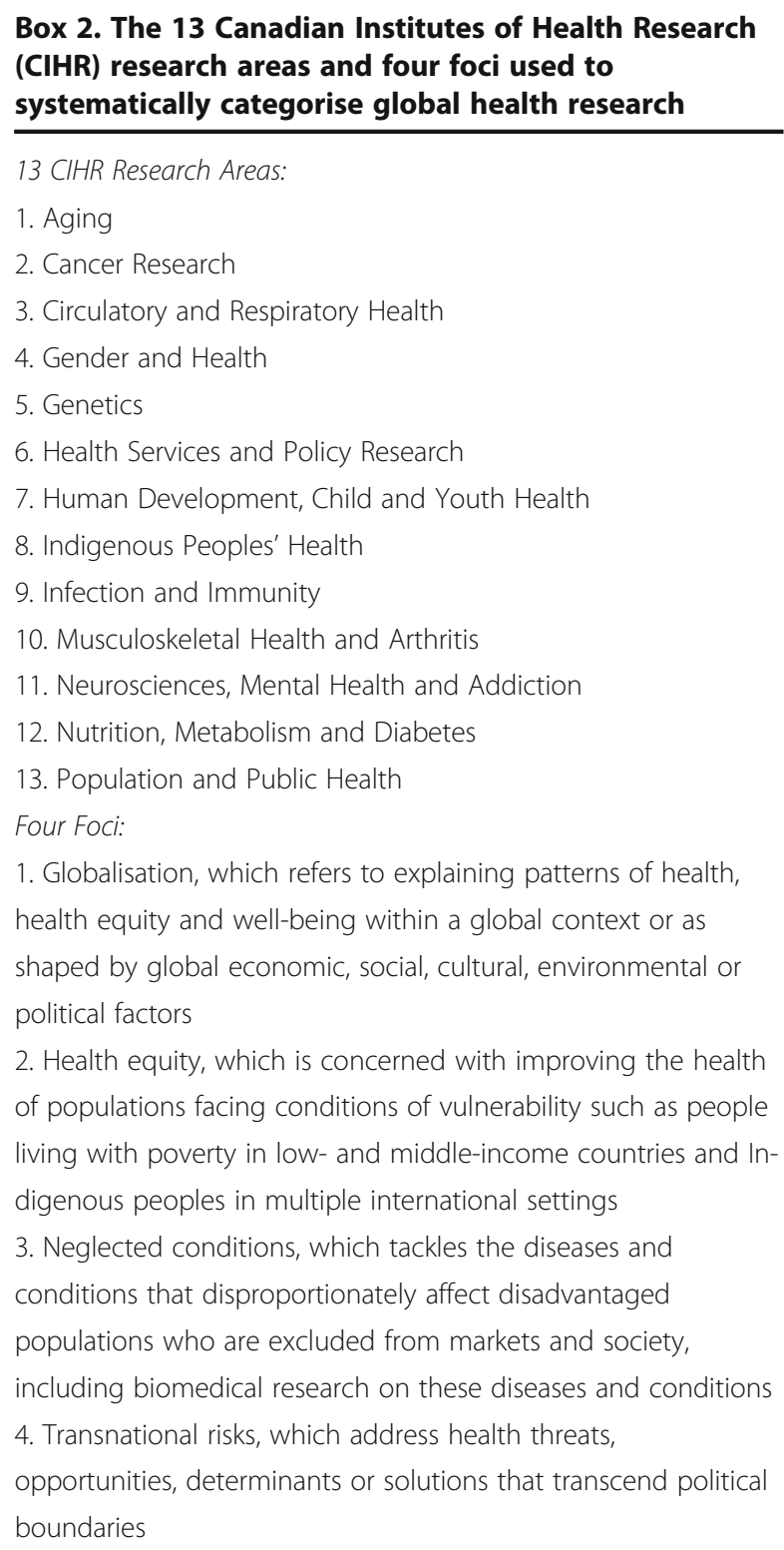

\section{Identifying research activities}

\section{Global health research training programmes}

We defined research training programmes as any research-focused graduate education programmes that explicitly focused on global health. An explicit focus on global health was indicated by the words 'global health' or 'international health' in the name of the programme, or any of its concentrations or specialisations. Indicators of a research-focused programme (as opposed to a professional focus) included a requirement or option to complete a thesis or major research project (as opposed to an internship, practicum or coursework-only programme).

We conducted three sets of systematic searches (Fig. 2) to identify global health research training programmes. The first systematic search focused on the main websites of the 30 most funded universities from our dataset of CIHR funding, while the second focused on the specific graduate studies websites of all 30 universities. Both types of websites were searched for the following terms: 'global health', 'international health', 'santé mondiale', 'santé internationale' and 'santé globale'. The first 100 results of each search were manually reviewed; this number was selected to balance the relevance of the result to the research question and screening feasibility. To supplement internet searches, a third systematic search used an administrative database on Canadian university programmes - the Universities Canada Database [15]. The aforementioned terms were used to search this database as well. The combined results of these searches were revisited and available programme descriptions were consulted to yield a list of global health research training programmes.

\section{Canada Research Chairs in Global Health}

The Canada Research Chairs (CRC) Program is a federal initiative that funds 2000 university professorships to attract and retain top research talent [16, 17]. We searched profiles in the CRCP Database for individuals conducting global health research using the following terms: 'global health', 'international health', 'population health' and 'health equity'. This database contained information such as the Chair's name, title, tier, host university, biography and research discipline.

\section{Research centres and WHO Collaborating Centres}

Global health research centres were defined as organised groups or units conducting research on global health that have been officially recognised as such by their host universities. We conducted two sets of systematic searches of the 30 universities' websites to identify relevant centres (Fig. 2). First, we searched the internal websites of these universities using the same key terms as our searches for research training programmes and reviewed the first 100 results. Second, we conducted an 


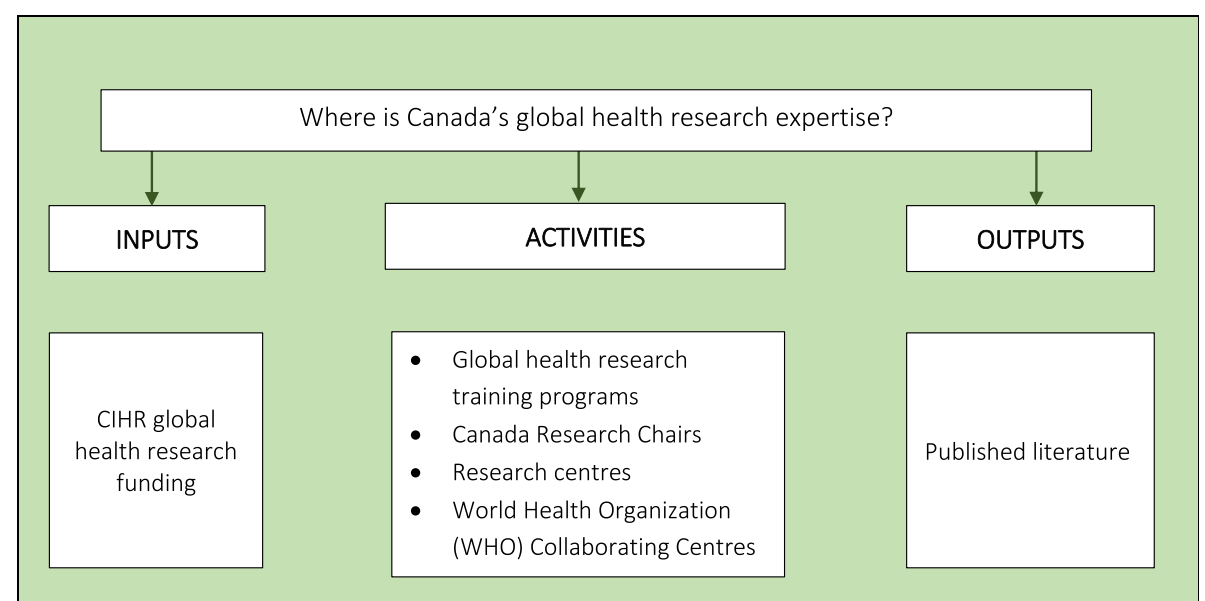

Fig. 1 Inputs, activities and outputs in global health research in the Canadian context

additional supplementary search whereby we sought out the 'Research' tab in the site navigation area of each university's website. This tab led to webpages that listed all research centres hosted by the university. We reviewed the lists available on these web pages to identify centres that conducted global health research. WHO Collaborating Centres are institutions or groups that conduct activities in support of WHO's various initiatives [18]. Designated by WHO's Director-General, Collaborating Centres can often partake in activities like research, training and education, advising WHO staff on specific topics, and collecting and collating information and resources [18]. We searched the WHO Collaborating Centres Database for information on the title of the centre, the designated parent institution (i.e. usually a university), the subjects of focus and the types of activities conducted [18, 19]. This information allowed us to understand which Canadian universities were involved and in what capacity, including which subject areas and what types of activities.

\section{Identifying research outputs \\ Prominent focus areas of publications}

We searched PubMed using a structured search query combining the Medical Subject Heading (MeSH) term 'Global Health' and Author Affiliation 'Canada' for articles published recently, from January 1, 2013, to March 1,2018 . We exported the search results and qualitatively coded abstracts to identify the foci of each global health research publication involving Canadian-affiliated authors as either as primary authors or as co-authors [7].

\section{Quantity of publications at 30 universities}

We conducted an additional search of PubMed for publications from April 1, 2000, to March 31, 2016, to identify the quantity of global health publications produced by authors affiliated with the 30 universities. We used this time period to match the fiscal-year funding period data provided by CIHR. In these searches, we used the Global Health MeSH term and, for the Affiliation field, we searched the names of each of the 30 universities.

\section{Results}

Our search shows that universities have developed their capacity in global health research to varying degrees. Canadian universities have a diverse range of global health research activity -12 global health research training programmes, 26 CRCs in global health, 6 research centres in global health and $30 \mathrm{WHO}$ collaborating centres (Table 1). Canadian global health research outputs include 822 unique citations with a Global Health MeSH in the PubMed database, with authors primarily belonging to universities in urban areas and research topics primarily focusing on 'health equity' and 'globalization'.

\section{Global health research training programmes}

We identified 12 global health research training programmes at 8 universities as described in Table 2; 4 universities - located in Toronto, Montreal, Alberta and London - hosted two programmes each, while the other 4 universities each hosted a single programme. Among the 12 programmes, 9 were Masters degree programmes, while 3 were doctoral programmes.

\section{Canada Research Chairs in Global Health}

Searching the CRC Database identified 26 individual chairs in different research disciplines at 10 universities and in 1 non-sample university (Table 3). Most CRCs in this search are at institutions in Montreal, Toronto, Vancouver and Ottawa, with 5 chairs in Montreal and 4 each in Toronto, Vancouver and Ottawa.

The majority of these CRCs conducted research that fits under CIHR's population health research pillar (65\%) and $38 \%$ specifically focused on globalisation. The 
Table 1 The 30 Canadian universities receiving the most Canadian Institutes of Health Research global health research funding

\begin{tabular}{|c|c|c|c|c|c|c|c|}
\hline \multirow{2}{*}{$\begin{array}{l}\text { Institution } \\
\text { name }\end{array}$} & \multirow[t]{2}{*}{ Location } & \multirow{2}{*}{$\begin{array}{l}\text { Input } \\
\text { Funding amount, } \\
\text { 2000-2016 } \\
(n=\$ 314,130,951)\end{array}$} & \multicolumn{4}{|l|}{ Activities } & \multirow{2}{*}{$\begin{array}{l}\text { Outputs } \\
\text { Publications with Global } \\
\text { Health MeSH }(n=1069)\end{array}$} \\
\hline & & & $\begin{array}{l}\text { Research training } \\
\text { programmes }(n= \\
\text { 12) }\end{array}$ & $\begin{array}{l}\text { Canada Research } \\
\text { Chairs in global health } \\
(n=26)\end{array}$ & $\begin{array}{l}\text { Research } \\
\text { centres } \\
(n=6)\end{array}$ & $\begin{array}{l}\text { WHO Collaborating } \\
\text { Centres }(n=30)\end{array}$ & \\
\hline $\begin{array}{l}\text { Acadia } \\
\text { University }\end{array}$ & Wolfville, NS & $\$ 196,000$ & 0 & 0 & 0 & 0 & 0 \\
\hline Brock University & $\begin{array}{l}\text { St. Catherine's, } \\
\text { ON }\end{array}$ & $\$ 157,285$ & 0 & 0 & 0 & 0 & 1 \\
\hline $\begin{array}{l}\text { Carleton } \\
\text { University }\end{array}$ & Ottawa, ON & $\$ 114,954$ & 0 & 0 & 0 & 0 & 5 \\
\hline $\begin{array}{l}\text { Dalhousie } \\
\text { University }\end{array}$ & Halifax, NS & $\$ 5,725,223$ & 0 & 4 & 0 & 1 & 32 \\
\hline $\begin{array}{l}\text { McGill } \\
\text { University }\end{array}$ & Montreal, QC & $\$ 47,881,835$ & 1 & 2 & 0 & 1 & 127 \\
\hline $\begin{array}{l}\text { McMaster } \\
\text { University }\end{array}$ & Hamilton, ON & $\$ 30,759,199$ & 1 & 1 & 0 & 2 & 128 \\
\hline $\begin{array}{l}\text { Queen's } \\
\text { University }\end{array}$ & Kingston, ON & $\$ 3,266,766$ & 0 & 0 & 0 & 0 & 28 \\
\hline $\begin{array}{l}\text { Ryerson } \\
\text { University }\end{array}$ & Toronto, ON & $\$ 609,017$ & 0 & 0 & 1 & 0 & 4 \\
\hline $\begin{array}{l}\text { Saint Mary's } \\
\text { University }\end{array}$ & Halifax, NS & $\$ 105,000$ & 0 & 3 & 0 & 0 & 1 \\
\hline $\begin{array}{l}\text { Simon Fraser } \\
\text { University }\end{array}$ & Burnaby, BC & $\$ 5,803,161$ & 1 & 1 & 0 & 0 & 31 \\
\hline $\begin{array}{l}\text { Université de } \\
\text { Montréal }\end{array}$ & Montreal, QC & $\$ 29,678,941$ & 2 & 0 & 0 & 0 & 28 \\
\hline $\begin{array}{l}\text { Université de } \\
\text { Sherbrooke }\end{array}$ & $\begin{array}{l}\text { Sherbrooke, } \\
\text { QC }\end{array}$ & $\$ 418,084$ & 0 & 0 & 0 & 1 & 2 \\
\hline $\begin{array}{l}\text { Université du } \\
\text { Québec }\end{array}$ & $\begin{array}{l}\text { Quebec City, } \\
\text { QC }\end{array}$ & $\$ 730,590$ & 0 & 0 & 0 & 0 & 4 \\
\hline $\begin{array}{l}\text { Université du } \\
\text { Québec à } \\
\text { Montréal }\end{array}$ & Montreal, QC & $\$ 667,703$ & 0 & 0 & 0 & 1 & 2 \\
\hline Université Laval & $\begin{array}{l}\text { Quebec City, } \\
\text { QC }\end{array}$ & $\$ 27,934,610$ & 1 & 0 & 0 & 0 & 16 \\
\hline $\begin{array}{l}\text { University of } \\
\text { Alberta }\end{array}$ & Edmonton, $\mathrm{AB}$ & $\$ 7,931,583$ & 2 & 0 & 0 & 1 & 71 \\
\hline $\begin{array}{l}\text { University of } \\
\text { British } \\
\text { Columbia }\end{array}$ & Vancouver, BC & $\$ 37,423,959$ & 0 & 4 & 1 & 1 & 111 \\
\hline $\begin{array}{l}\text { University of } \\
\text { Calgary }\end{array}$ & Calgary, AB & $\$ 4,175,934$ & 0 & 0 & 0 & 1 & 60 \\
\hline $\begin{array}{l}\text { University of } \\
\text { Guelph }\end{array}$ & Guelph, ON & $\$ 534,900$ & 0 & 0 & 0 & 0 & 7 \\
\hline $\begin{array}{l}\text { University of } \\
\text { Lethbridge }\end{array}$ & Lethbridge, $A B$ & $\$ 100,000$ & 0 & 2 & 1 & 0 & 4 \\
\hline $\begin{array}{l}\text { University of } \\
\text { Manitoba }\end{array}$ & Winnipeg, MB & $\$ 24,044,245$ & 0 & 4 & 0 & 0 & 31 \\
\hline $\begin{array}{l}\text { University of } \\
\text { Ottawa }\end{array}$ & Ottawa, ON & $\$ 14,969,455$ & 0 & 2 & 1 & 1 & 99 \\
\hline $\begin{array}{l}\text { University of } \\
\text { Prince Edward } \\
\text { Island }\end{array}$ & $\begin{array}{l}\text { Charlottetown, } \\
\text { PEI }\end{array}$ & $\$ 168,750$ & 0 & 1 & 0 & 0 & 0 \\
\hline $\begin{array}{l}\text { University of } \\
\text { Regina }\end{array}$ & Regina, SK & $\$ 1,208,518$ & 0 & 0 & 0 & 0 & 3 \\
\hline $\begin{array}{l}\text { University of } \\
\text { Saskatchewan }\end{array}$ & Saskatoon, SK & $\$ 3,646,490$ & 0 & 0 & 0 & 0 & 17 \\
\hline University of & Toronto, ON & $\$ 48,370,150$ & 2 & 4 & 1 & 4 & 279 \\
\hline
\end{tabular}


Table 1 The 30 Canadian universities receiving the most Canadian Institutes of Health Research global health research funding (Continued)

\begin{tabular}{|c|c|c|c|c|c|c|c|}
\hline \multirow{2}{*}{$\begin{array}{l}\text { Institution } \\
\text { name }\end{array}$} & \multirow[t]{2}{*}{ Location } & \multirow{2}{*}{$\begin{array}{l}\text { Input } \\
\text { Funding amount, } \\
2000-2016 \\
(n=\$ 314,130,951)\end{array}$} & \multicolumn{4}{|l|}{ Activities } & \multirow{2}{*}{$\begin{array}{l}\text { Outputs } \\
\text { Publications with Global } \\
\text { Health MeSH ( } n=1069)\end{array}$} \\
\hline & & & $\begin{array}{l}\text { Research training } \\
\text { programmes }(n= \\
\text { 12) }\end{array}$ & $\begin{array}{l}\text { Canada Research } \\
\text { Chairs in global health } \\
(n=26)\end{array}$ & $\begin{array}{l}\text { Research } \\
\text { centres } \\
(n=6)\end{array}$ & $\begin{array}{l}\text { WHO Collaborating } \\
\text { Centres }(n=30)\end{array}$ & \\
\hline \multicolumn{8}{|l|}{ Toronto } \\
\hline $\begin{array}{l}\text { University of } \\
\text { Victoria }\end{array}$ & Victoria, BC & $\$ 2,354,077$ & 0 & 0 & 0 & 0 & 14 \\
\hline $\begin{array}{l}\text { University of } \\
\text { Waterloo }\end{array}$ & Kitchener, ON & $\$ 12,090,327$ & 0 & 0 & 0 & 0 & 12 \\
\hline $\begin{array}{l}\text { University of } \\
\text { Western } \\
\text { Ontario }\end{array}$ & London, ON & $\$ 2,308,191$ & 2 & 0 & 1 & 0 & 17 \\
\hline York University & Toronto, ON & $\$ 756,000$ & 0 & 0 & 1 & 0 & 62 \\
\hline
\end{tabular}

Funding data obtained from Canadian Institutes of Health Research. Publication counts include duplicates whenever records were co-authored by researchers at more than one of the 30 universities; there were 822 unique publications

A. Search strategy used to identify research training programs in global health

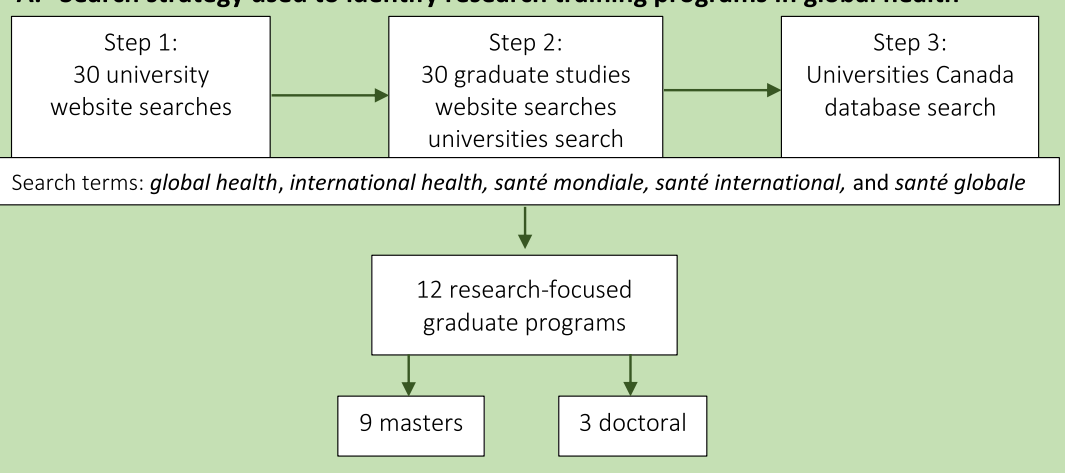

B. Search strategy to identify Canada Research Chairs in Global Health

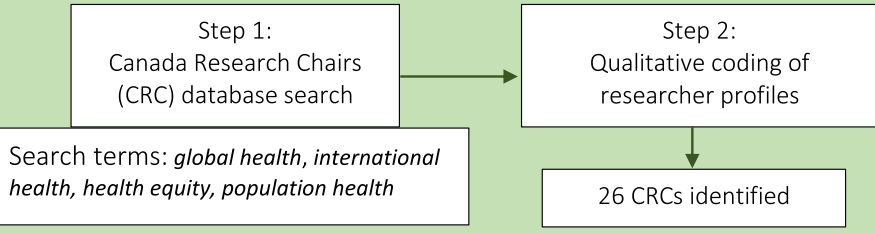

C. Search strategy to identify research centres in global health

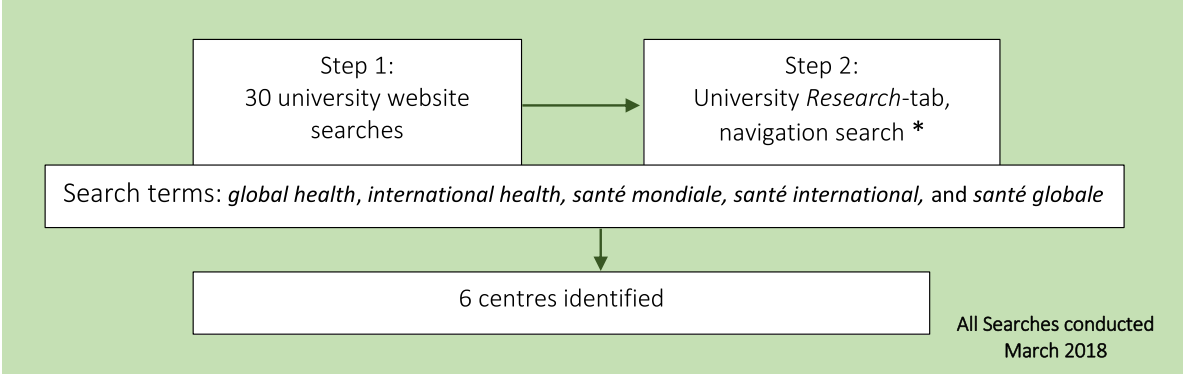

* This step was not conducted when the institution website was missing a Research tab or related section in their sitemap.

Fig. 2 Search strategies used to identify research training programmes, Canada Research Chairs in global health and research centres. *This step was not conducted when the institution website was missing a Research tab or related section in their sitemap 
Table 2 Global health research training programmes and research centres in Canada

\begin{tabular}{|c|c|c|c|}
\hline \multicolumn{4}{|l|}{ Research training programmes } \\
\hline Programme title & Programme level & University & Location \\
\hline MSc in Public Health (Global Health Option) & Masters & McGill University & Montreal, QC \\
\hline MSc in Global Health & Masters & McMaster University & Hamilton, ON \\
\hline MSc in Global Health & Masters & Simon Fraser University & Burnaby, BC \\
\hline MSc en santé publique (option de santé mondiale) & Masters & Université de Montréal & Montreal, QC \\
\hline MPH en santé mondiale & Masters & Université Laval & $\begin{array}{l}\text { Quebec City, } \\
\text { QC }\end{array}$ \\
\hline MPH in Global Health & Masters & University of Alberta & Edmonton, $\mathrm{AB}$ \\
\hline MSc in Global Health & Masters & University of Alberta & Edmonton, $\mathrm{AB}$ \\
\hline Masters Collaborative Specialization in Global Health & Masters & University of Toronto & Toronto, ON \\
\hline $\begin{array}{l}\text { Masters Collaborative Program in Global Health Systems in } \\
\text { Africa }\end{array}$ & Masters & $\begin{array}{l}\text { University of Western } \\
\text { Ontario }\end{array}$ & London, ON \\
\hline PhD en santé publique (de l'option "santé mondiale") & Doctoral & Université de Montréal & Montreal, QC \\
\hline Doctoral Collaborative Specialization in Global Health & Doctoral & University of Toronto & Toronto, ON \\
\hline $\begin{array}{l}\text { Doctoral Collaborative Program in Global Health Systems in } \\
\text { Africa }\end{array}$ & Doctoral & $\begin{array}{l}\text { University of Western } \\
\text { Ontario }\end{array}$ & London, ON \\
\hline \multicolumn{4}{|l|}{ Research centres } \\
\hline Title & Director(s) & University & Location \\
\hline Centre for Global Health and Health Equity & $\begin{array}{l}\text { Vahabi, Mandana \& Guruge, } \\
\text { Sepali }\end{array}$ & Ryerson University & Toronto, ON \\
\hline Global Health Research Program & Spiegel, Jerry \& Yassi, Annalee & $\begin{array}{l}\text { University of British } \\
\text { Columbia }\end{array}$ & Vancouver, BC \\
\hline Centre for Global Public Health & Blanchard, James & University of Manitoba & Winnipeg, MB \\
\hline Centre for Global Health & Tugwell, Peter & University of Ottawa & Ottawa, ON \\
\hline Institute for Global Health Equity and Innovation & Jadad, Alex & University of Toronto & Toronto, ON \\
\hline Dahdaleh Institute for Global Health Research & Orbinski, James & York University & Toronto, ON \\
\hline
\end{tabular}

Data retrieved from institution website searches and database searches in March 2018

programme supports both "exceptional emerging researchers" and "outstanding established researchers", given the Tier 2 and Tier 1 title, respectively [16]. More than half are Tier 2 CRCs (58\%), while the remaining $42 \%$ are Tier 1 CRCs. Women made up 34\% $(n=9)$ of the CRCs in global health.

\section{Research centres and WHO Collaborating Centres}

There are 6 officially recognised groups dedicated to conducting global health research (Table 2) and 30 actively designated WHO Collaborating Centres (Table 4). As of March 2018, the city of Toronto hosted the greatest number of both research centres and WHO Collaborating Centres. Canadian WHO Collaborating Centres are mostly engaged in training and education, product development (e.g. guidelines, manuals, methodologies) and research (Table 5). The substantive issues being addressed in the Collaborating Centres vary; however, the most popular issues include environmental health and hazards (6 of 30 centres) and occupational health (6 of 30 centres).

\section{Prominent focus areas of publications}

Overall, 822 unique publications were retrieved from the PubMed search covering January 1, 2013, to March 1, 2018. Of the 822 publications, 249 focused on globalisation and 244 focused on health equity. Transnational risks and neglected conditions followed with 90 and 58 publications, respectively, with an additional 183 publications coded as other. Specific research topics within these categories varied greatly. For example, the globalisation publications looked at issues like global health governance, North-South development partnerships, trade agreements and multilateral organisations [20-22]. Within health equity, publications were focused on issues pertaining to child and maternal health, with research involving sex workers, adolescents and Indigenous peoples appearing less often [23, 24]. Publications on neglected conditions included studies on malaria, polio and tuberculosis in LMIC contexts, while publications on transnational risks looked at infectious diseases with pandemic potential [25]. 
Table 3 Canada Research Chairs in global health

\begin{tabular}{|c|c|c|c|c|c|}
\hline Chairholder & Chair title & Tier & University & Recruited from & Research foci \\
\hline 1. Brockman, Mark & Viral Pathogenesis and Immunity & 2 & Simon Fraser University & Harvard Medical School, USA & $\begin{array}{l}\text { Neglected } \\
\text { Conditions }\end{array}$ \\
\hline 2. Chan, Laurie & $\begin{array}{l}\text { Toxicology and Environmental } \\
\text { Health }\end{array}$ & 1 & University of Ottawa & Domestic & Globalisation \\
\hline 3. Forman, Lisa & $\begin{array}{l}\text { Human Rights and Global Health } \\
\text { Equity }\end{array}$ & 2 & University of Toronto & Domestic & Health equity \\
\hline 4. Frank, Erica & $\begin{array}{l}\text { Preventive Medicine and } \\
\text { Population Health }\end{array}$ & 1 & $\begin{array}{l}\text { University of British } \\
\text { Columbia }\end{array}$ & Emory University School of Medicine & Other \\
\hline $\begin{array}{l}\text { 5. Grimshaw, } \\
\text { Jeremy }\end{array}$ & $\begin{array}{l}\text { Health Knowledge Transfer and } \\
\text { Uptake }\end{array}$ & 1 & University of Ottawa & University of Aberdeen, UK & Other \\
\hline 6. Jha, Prabhat & Global Health & 1 & University of Toronto & Domestic & Other \\
\hline 7. Kaufman, Jay & Health Disparities & 1 & McGill University & $\begin{array}{l}\text { University of North Carolina at Chapel } \\
\text { Hill, USA }\end{array}$ & Globalisation \\
\hline $\begin{array}{l}\text { 8. Labonté, } \\
\text { Ronald }\end{array}$ & Globalization and Health Equity & 1 & University of Ottawa & Domestic & Globalisation \\
\hline 9. Lee, Kelley & Global Health Governance & 1 & Simon Fraser University & $\begin{array}{l}\text { London School of Hygiene \& Tropical } \\
\text { Medicine, UK }\end{array}$ & Globalisation \\
\hline 10. Love, Oliver & Integrative Ecology & 2 & University of Windsor & Domestic & Globalisation \\
\hline $\begin{array}{l}\text { 11. Masuda, } \\
\text { Jeffrey }\end{array}$ & Environmental Health Equity & 2 & Queen's University & Domestic & Globalisation \\
\hline 12. Menec, Verena & Healthy Aging & 2 & University of Manitoba & Domestic & Health equity \\
\hline 13. Nandi, Arijit & $\begin{array}{l}\text { Political Economy of Global } \\
\text { Health }\end{array}$ & 2 & McGill University & Harvard University, USA & Globalisation \\
\hline $\begin{array}{l}\text { 14. Nicolau, } \\
\text { Belinda }\end{array}$ & Life Course Oral Epidemiology & 2 & McGill University & Domestic & Health equity \\
\hline 15. Price, Eric & Radiochemistry & 2 & $\begin{array}{l}\text { University of } \\
\text { Saskatchewan }\end{array}$ & $\begin{array}{l}\text { Memorial Sloan Kettering Cancer Center, } \\
\text { USA }\end{array}$ & $\begin{array}{l}\text { Neglected } \\
\text { conditions }\end{array}$ \\
\hline $\begin{array}{l}\text { 16. Revie, } \\
\text { Crawford }\end{array}$ & Population Health: Epi-Informatics & 2 & $\begin{array}{l}\text { University of Prince } \\
\text { Edward Island }\end{array}$ & University of Strathclyde, UK & $\begin{array}{l}\text { Transnational } \\
\text { risks }\end{array}$ \\
\hline 17. Rosella, Laura & Population Health Analytics & 2 & University of Toronto & Domestic & $\begin{array}{l}\text { Neglected } \\
\text { conditions }\end{array}$ \\
\hline 18. Sagan, Selena & RNA Biology and Viral Infections & 2 & McGill University & Stanford University, USA & $\begin{array}{l}\text { Neglected } \\
\text { conditions }\end{array}$ \\
\hline $\begin{array}{l}\text { 19. Schechter, } \\
\text { Martin T. }\end{array}$ & $\begin{array}{l}\text { HIV/AIDS and Urban Population } \\
\text { Health }\end{array}$ & 1 & $\begin{array}{l}\text { University of British } \\
\text { Columbia }\end{array}$ & Domestic & $\begin{array}{l}\text { Neglected } \\
\text { conditions }\end{array}$ \\
\hline 20. Sin, Don D. & $\begin{array}{l}\text { Chronic Obstructive Pulmonary } \\
\text { Disease }\end{array}$ & 1 & $\begin{array}{l}\text { University of British } \\
\text { Columbia }\end{array}$ & Domestic & Other \\
\hline 21. Tugwell, Peter & Health Equity & 1 & University of Ottawa & Domestic & Globalisation \\
\hline $\begin{array}{l}\text { 22. Urquia, } \\
\text { Marcelo }\end{array}$ & Applied Population Health & 2 & University of Manitoba & Domestic & Globalisation \\
\hline $\begin{array}{l}\text { 23. Vallée-Bélisle, } \\
\text { Alexis }\end{array}$ & $\begin{array}{l}\text { Bioengineering and } \\
\text { Bionanotechnology }\end{array}$ & 2 & Université de Montréal & Domestic & Other \\
\hline $\begin{array}{l}\text { 24. Waddell, } \\
\text { Charlotte }\end{array}$ & Children's Health Policy & 2 & Simon Fraser University & Domestic & Globalisation \\
\hline 25. Winer, Daniel & Immunometabolism & 2 & University of Toronto & Domestic & Other \\
\hline 26. Yassi, Annalee & $\begin{array}{l}\text { Global Health and Capacity } \\
\text { Building }\end{array}$ & 1 & $\begin{array}{l}\text { University of British } \\
\text { Columbia }\end{array}$ & Domestic & Other \\
\hline
\end{tabular}

Data retrieved from the CRC Database in March 2018

\section{Authors with Canadian affiliations and quantity of publications at 30 universities}

We identified the 30 most frequently published Canadian-affiliated researchers among articles classified under the Global Health MeSH (Table 6). These 30 researchers were primary authors or co-authors on at least four or more of the 822 publications; 3 of 26 CRCs also appeared on this list. The second PubMed search of 
Table 4 WHO Collaborating Centres in Canada

\begin{tabular}{|c|c|c|c|}
\hline WHO Collaborating Centre in... & Director & Host institution & Location \\
\hline 1. Addiction and Mental Health & Shield, Kevin & Centre for Addiction and Mental Health & Toronto, ON \\
\hline 2. Age-friendly Cities and Communities & Garon, Suzanne & Centre hospitalier universitaire de Sherbrooke & $\begin{array}{l}\text { Sherbrooke, } \\
\text { QC }\end{array}$ \\
\hline 3. Bioethics & Gibson, Jennifer & University of Toronto & Toronto, ON \\
\hline 4. Biosafety and Biosecurity & Mantha, Stacey & Public Health Agency of Canada & Ottawa, ON \\
\hline 5. Classification, Terminology and Standards & Quan, Hude & University of Calgary & Calgary, AB \\
\hline 6. Control and Epidemiology of Rabies in Carnivores & $\begin{array}{l}\text { Fehlner-Gardiner, } \\
\text { Christine }\end{array}$ & Canadian Food Inspection Agency & Nepean, ON \\
\hline 7. Evidence-Informed Policy & Lavis, John N. & McMaster University & $\begin{array}{l}\text { Hamilton, } \\
\text { ON }\end{array}$ \\
\hline $\begin{array}{l}\text { 8. Governance, Transparency and Accountability in the } \\
\text { Pharmaceutical Sector }\end{array}$ & Kohler, Jillian & University of Toronto & Toronto, ON \\
\hline 9. Health Promotion & Jackson, Suzanne & University of Toronto & Toronto, ON \\
\hline $\begin{array}{l}\text { 10. Knowledge Translation and Health Technology } \\
\text { Assessment in Health Equity }\end{array}$ & $\begin{array}{l}\text { Hatcher-Roberts, } \\
\text { Janet }\end{array}$ & University of Ottawa & Ottawa, ON \\
\hline 11. Monitoring Chemical Contaminants in Food & Feeley, Mark & Health Canada & Ottawa, ON \\
\hline 12. Nutrition Policy for Chronic Disease Prevention & L'Abbe, Mary & University of Toronto & Toronto, ON \\
\hline 13. Occupational and Environmental Health & $\begin{array}{l}\text { Saint-Charles, } \\
\text { Johanne }\end{array}$ & Université du Québec à Montréal & $\begin{array}{l}\text { Montréal, } \\
\text { QC }\end{array}$ \\
\hline 14. Occupational Health and Safety & Jones, Gareth & Canadian Centre for Occupational Health \& Safety & $\begin{array}{l}\text { Hamilton, } \\
\text { ON }\end{array}$ \\
\hline 15. Patient Safety and Patient Engagement & Kossey, Sandi & Canadian Patient Safety Institute & $\begin{array}{l}\text { Edmonton, } \\
A B\end{array}$ \\
\hline 16. Research and Training in Mental Health & Laporta, Marc & McGill University & $\begin{array}{l}\text { Montréal, } \\
\text { QC }\end{array}$ \\
\hline $\begin{array}{l}\text { 17. Research and Training in Parasite Epidemiology and } \\
\text { Control }\end{array}$ & Gyorkos, Theresa & McGill University & $\begin{array}{l}\text { Montréal, } \\
\text { QC }\end{array}$ \\
\hline 18. Safety Promotion and Injury Prevention & Maurice, Pierre & Centre de Santé publique du Québec & $\begin{array}{l}\text { Beauport, } \\
\text { QC }\end{array}$ \\
\hline 19. Standardization and Evaluation of Biologicals & Elmgren, Lindsay & Health Canada & Ottawa, ON \\
\hline 20. Studying Peri-operative Surgical Care & Cheng, Davy & University of Western Ontario & London, ON \\
\hline 21. Water Quality & Carreau, Greg & Health Canada & Ottawa, ON \\
\hline 22. Occupational and Environmental Health & Yassi, Annalee & University of British Columbia & $\begin{array}{l}\text { Vancouver, } \\
\text { BC }\end{array}$ \\
\hline 23. Primary Care Nursing and Health Human Resources & Baumann, Andrea & McMaster University & $\begin{array}{l}\text { Hamilton, } \\
\text { ON }\end{array}$ \\
\hline 24. Children's Environmental Health & Buka, Irena & University of Alberta & $\begin{array}{l}\text { Edmonton, } \\
A B\end{array}$ \\
\hline $\begin{array}{l}\text { 25. Environmental and Occupational Health Impact } \\
\text { Assessment and Surveillance }\end{array}$ & Gosselin, Pierre & Centre Hospitalier Universitaire de Québec & $\begin{array}{l}\text { Québec } \\
\text { City, QC }\end{array}$ \\
\hline 26. Health Science Education and Practice & Morin, Martine & Université de Sherbrooke & $\begin{array}{l}\text { Sherbrooke, } \\
\text { QC }\end{array}$ \\
\hline 27. Health Workforce Planning and Research & Murphy, Gail T. & Dalhousie University & Halifax, NS \\
\hline 28. Non-Communicable Disease Policy & Rodin, Rachel & Public Health Agency of Canada & Ottawa, ON \\
\hline 29. Occupational and Environmental Cancer & Demers, Paul & Cancer Care Ontario & Toronto, ON \\
\hline 30. Occupational Health & Lazure, Louis & $\begin{array}{l}\text { Institut de recherche Robert-Sauvé en santé et en } \\
\text { sécurité du travail }\end{array}$ & $\begin{array}{l}\text { Montréal, } \\
\text { QC }\end{array}$ \\
\hline
\end{tabular}

Data retrieved from the WHO Collaboration Centre Database in March 2018 
Table 5 Types of activities conducted and subjects studied by the 30 active WHO Collaborating Centres in Canada

\begin{tabular}{|c|c|}
\hline $\begin{array}{l}\text { Type of activity conducted by WHO } \\
\text { Collaborating Centres }\end{array}$ & $\begin{array}{l}\text { Number of centres } \\
\text { conducting activity }(n=30)\end{array}$ \\
\hline Training and education & 23 \\
\hline $\begin{array}{l}\text { Product development (e.g. guidelines, } \\
\text { manuals, protocols) }\end{array}$ & 14 \\
\hline Research & 10 \\
\hline $\begin{array}{l}\text { Collection and collation of } \\
\text { information }\end{array}$ & 9 \\
\hline Providing technical advice to $\mathrm{WHO}$ & 9 \\
\hline Information dissemination & 6 \\
\hline $\begin{array}{l}\text { Coordination of activities carried out } \\
\text { by several institutions (e.g. Other WHO } \\
\text { collaborating centres) }\end{array}$ & 4 \\
\hline $\begin{array}{l}\text { Implementation of WHO programmes } \\
\text { and activities at country level }\end{array}$ & 3 \\
\hline $\begin{array}{l}\text { Development and application of } \\
\text { appropriate technology }\end{array}$ & 2 \\
\hline $\begin{array}{l}\text { Organisation of events (e.g. } \\
\text { conferences, summits) }\end{array}$ & 2 \\
\hline Subject & $\begin{array}{l}\text { Number of centres } \\
\text { studying subject }(n=30)\end{array}$ \\
\hline $\begin{array}{l}\text { Environmental health and hazards } \\
\text { other than those specifically } \\
\text { mentioned }\end{array}$ & 6 \\
\hline Occupational health & 6 \\
\hline $\begin{array}{l}\text { Health information, statistics, } \\
\text { measurement and trend assessment }\end{array}$ & 4 \\
\hline $\begin{array}{l}\text { Human resources for health } \\
\text { (excluding nursing) }\end{array}$ & 4 \\
\hline Cardiovascular diseases & 3 \\
\hline $\begin{array}{l}\text { Health systems research and } \\
\text { development }\end{array}$ & 3 \\
\hline Research policy and development & 3 \\
\hline Biological & 2 \\
\hline Chemical safety & 2 \\
\hline Ethics & 2 \\
\hline
\end{tabular}

Data retrieved from WHO Collaborating Centres Database in March 2018. Activity classifications and subject classifications were obtained from the WHO Collaborating Centre Database entries for each centre

published literature yielded a total of 1069 citations. Each of these citations has at least 1 author with an affiliation at 1 of the 30 universities and the search showed that university-affiliated researchers that were situated in urban areas (Toronto and Montreal) produced the most publications classified with the Global Health $\mathrm{MeSH}$ term.

\section{Discussion}

This study developed a three-pronged approach to map a country's global health research strengths and expertise using research inputs, activities and outputs. The rapid environmental scan employed publicly available administrative datasets to paint a picture of the current landscape of Canada's global health research expertise. Methods such as these can help shape policy when there is an urgent or time-sensitive need to assess strengths using an efficient, yet simple and resource-friendly approach. Our findings can continue supporting strategic and evidence-informed funding policies for Canada's global health research stakeholders.

\section{Global health research in Canada}

Our searches show that the main global health research strengths in Canada are health equity and globalisation. Health equity was the most common focus of research funded by CIHR grants. This research tended to focus on maternal and child health in LMICs and reflects Canada's international development assistance priorities over the past decade [6]. Globalisation also emerged as the most common focus of CRC research interests and the focus of publications captured in the PubMed search. Future studies should explore this finding in greater detail.

Our approach suggests that Canada's global health research expertise may be highly concentrated within a few urban areas. Based on the measures used in this scan, global health research activity appears to be flourishing in Toronto and Montreal, with the strongest contributions from 2 universities (Fig. 3). These 2 particular universities received the most CIHR global health research funding, which translated to the greatest global health research activity out of the 30 universities. We hypothesise that urban regions generally - and these universities in particular - may be benefiting from a positive feedback loop involving higher research funding levels, more CRCs, and increased research training opportunities and reputations for overall research excellence. The benefits of being situated in urban areas may include larger networks of researchers and society/government partners and better access to physical resources like high-containment biohazard labs and large teaching hospitals.

While global health research expertise is concentrated in Toronto and Montreal, at least some expertise is found in nearly all research-intensive universities across the country. Specifically, 27 of 30 universities studied here are involved in global health research through different combinations of activities. Interestingly, some universities had research centres devoted specifically to global health, but we could not identify any global health training programmes. Alternatively, universities can be less engaged with global health research training programmes, CRCs and published research contributions, while still hosting WHO Collaborating Centres. 


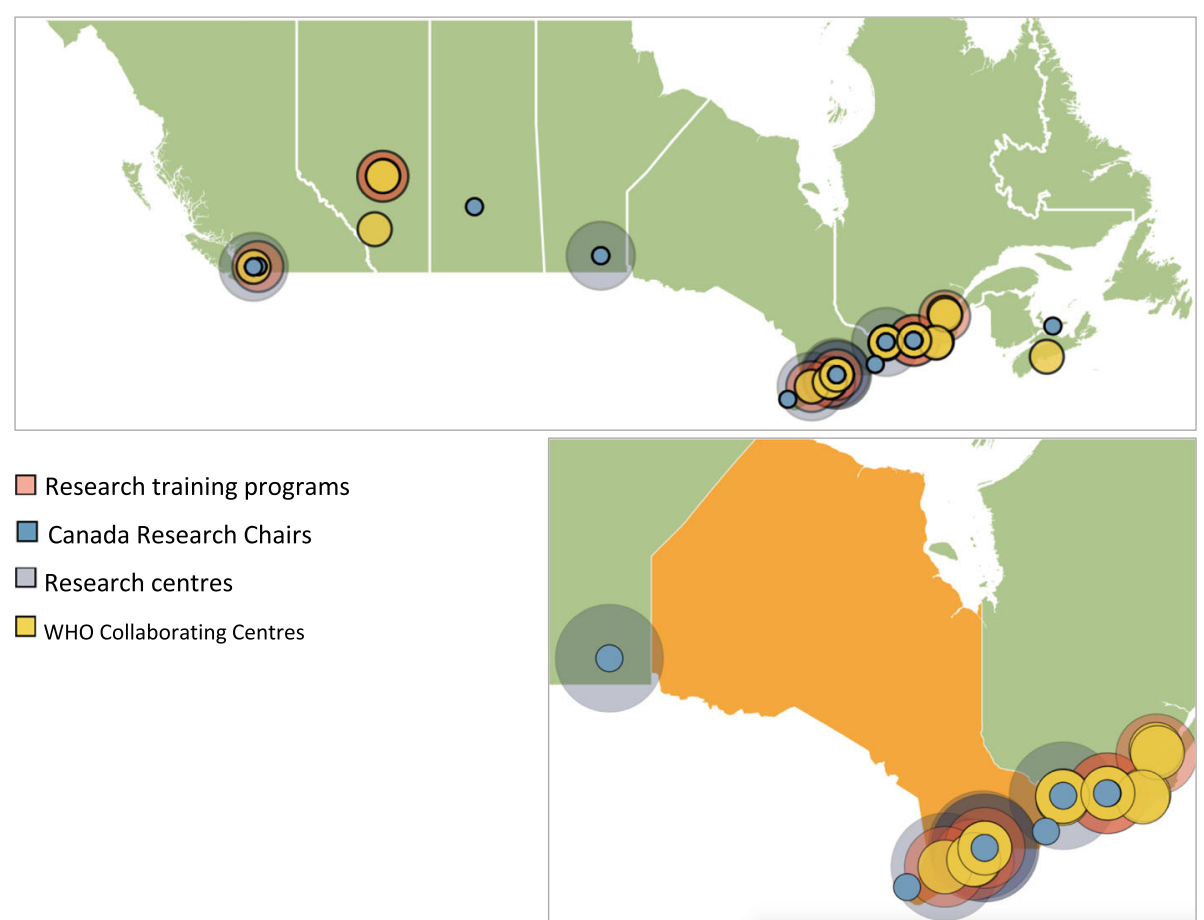

Fig. 3 Map showing the concentration of global health research training programmes, Canada Research Chairs, research centres and WHO Collaborating Centres across Canada

\section{Research and policy implications \\ The need for a standard and operational definition of 'global health research'}

Our research highlights the needs for a more consistent and operational definition of global health research. For example, we did not capture the full extent of research on the VSV-EBOV Ebola vaccine deployed in the emergency response to the March 2016 outbreak in our CRC and PubMed searches. This discrepancy may point to a divergence between the definitional scope of global health research and those researchers who either use this term, identify themselves as part of this epistemic community or actively participate within it. For example, whereas global health researchers may consider the developers of the Ebola vaccine to be global health researchers, Ebola vaccine researchers may not - instead considering themselves as infectious disease researchers, vaccinologists or virologists - and as a result may not be members of leading organisations of Canadian global health researchers (i.e. Canadian Coalition for Global Health Research) or attendees of the field's conference (i.e. Canadian Conference on Global Health). One example of this discrepancy is the CIHR-funded CRC in Molecular Virology and Antiviral Therapeutics, Marceline Côté, whose CRC profile states that their chemical genomics and genetics research "supports the development of antiviral therapies to fight emerging viruses, such as Ebola, and will help train the next generation of virology experts" [26]. 'Global health', or 'international health', is not mentioned in their profile nor was their name captured in the CRC database search, but some may argue that clinical work on a transnational virus like Ebola would allow individuals to identify as 'global health' researchers. A standard and operational definition of global health research can help address the challenges we face in accurately mapping global health research expertise and support our quest for robust capacity-building.

\section{Feasibly identifying a country's global health research expertise}

This method proved feasible and efficient for identifying a country's research expertise. It relies on a logical framework of inputs, activities and outputs, and employs publicly available administrative datasets to identify the geographic and substantive foci of significant global health research expertise. In applying this method, it is clear that Canada has unique strengths and our findings have informed CIHR's development of a strategic plan. In a tangible sense, these methods can be adapted and used to support research policy directives, including to inform national global health research strategies and to allow universities, civil society and research funding organisations to make more strategic decisions regarding their respective priorities and mandates. 
Table 6 The 30 authors with the greatest quantity of publications coded with the Global Health MeSH and Canadian affiliations from January 1, 2013, to March 1, 2018

\begin{tabular}{|c|c|c|c|c|}
\hline $\begin{array}{l}\text { Publications } \\
(n=822)\end{array}$ & Author's Name & $\begin{array}{l}\text { Author's } \\
h \text {-index }\end{array}$ & Author's institutional affiliation (as listed on Scopus) & Location \\
\hline 11 & Rehm J & 14 & Centre for Addiction and Mental Health & Toronto, ON \\
\hline 9 & Yusuf $\mathrm{S}$ & 193 & Population Health Research Institute & Hamilton, ON \\
\hline 8 & Lee $\mathrm{K}$ & 27 & Simon Fraser University, Faculty of Health Sciences & Burnaby, BC \\
\hline 7 & Armstrong PW & 100 & University of Alberta, Department of Medicine & Edmonton, $\mathrm{AB}$ \\
\hline 7 & Labonté R & 35 & University of Ottawa, School of Public Health and Preventive Medicine & Ottawa, ON \\
\hline 6 & Cole DC & 40 & University of Toronto, Dalla Lana School of Public Health & Toronto, ON \\
\hline 6 & Forman $L$ & 9 & University of Toronto, Dalla Lana School of Public Health & Toronto, ON \\
\hline 6 & Grace SL & 34 & York University & Vancouver, BC \\
\hline 6 & Hoffman SJ & 16 & York University, Faculty of Health and Osgoode Hall Law School & Ottawa, ON \\
\hline 6 & Lencucha R & 10 & McGill University, Faculty of Medicine & Montreal, QC \\
\hline 6 & Raina P & 39 & McMaster University, McMaster Evidence Review and Synthesis Centre & Hamilton, ON \\
\hline 6 & Ruckert A & 10 & University of Ottawa, School of Public Health and Preventive Medicine & Ottawa, ON \\
\hline 5 & Ali $U$ & 8 & McMaster University, Department of Clinical Epidemiology and Biostatistics & Hamilton, ON \\
\hline 5 & Balion C & 19 & McMaster University, Faculty of Health Sciences, Department of Medicine & Hamilton, ON \\
\hline 5 & Brown JA & 13 & McMaster University, Program in Evidence-Based Care & Hamilton, ON \\
\hline 5 & Bustamam A & 8 & McMaster University, Department of Clinical Epidemiology and Biostatistics & Hamilton, ON \\
\hline 5 & Clark J & 4 & University of Toronto, Department of Medical Imaging & Toronto, ON \\
\hline 5 & Khan K & 27 & Li Ka Shing Knowledge Institute & Toronto, ON \\
\hline 5 & McKelvie R & 61 & The University of Western Ontario & London, ON \\
\hline 5 & Oremus M & 18 & University of Waterloo, School of Public Health and Health Systems & Waterloo, ON \\
\hline 5 & Sohel N & 14 & McMaster University, Department of Clinical Epidemiology and Biostatistics ${ }^{a}$ & Hamilton, ON \\
\hline 4 & Atallah R & 7 & Jewish General Hospital, Division of Clinical Epidemiology & Montreal, QC \\
\hline 4 & Booth RA & 15 & University of Ottawa, Canada, Faculty of Medicine & Ottawa, ON \\
\hline 4 & Campbell NR & 47 & University of Calgary, Department of Medicine & Ottawa, ON \\
\hline 4 & Eisenberg MJ & 59 & McGill University & Montreal, QC \\
\hline 4 & Ezekowitz JA & 45 & University of Alberta, Canadian VIGOUR Centre & Edmonton, $\mathrm{AB}$ \\
\hline 4 & Goodman SG & 71 & University of Toronto, St. Michael's Hospital, Department of Medicine & Toronto, ON \\
\hline 4 & Hill SA & 24 & Hamilton Health Sciences & Hamilton, ON \\
\hline 4 & Kaplan GG & 40 & University of Calgary, Inflammatory Bowel Disease Clinic & Calgary, AB \\
\hline 4 & Krahn AD & 62 & University of British Columbia, Heart Rhythm Services & Vancouver, BC \\
\hline
\end{tabular}

Information retrieved from the PubMed and Scopus databases

${ }^{\text {a }}$ Sohel $\mathrm{N}$ did not have an affiliation listed in the Scopus database, so it was instead identified through one of their recent publications listed in PubMed

\section{Strengths and limitations}

This rapid environmental scan methodology allowed us to summarise existing data on Canada's global health research expertise. Strengths of the method include its careful integration of a logical framework (one that considers a variety of inputs, activities and outputs), the use of publicly available data, and both methodological and data triangulation. The logical framework ensures that research inputs can be systematically attributed to research activities and research outputs that are relevant to each individual country. Using publicly available data reduces the barriers that arise from a lack of access. For example, other inputs include grants from industry yet, due to privacy laws, this type of information is not available publicly. The methodological triangulation and data triangulation ensure built-in redundancies that can help verify and challenge findings.

A limitation of this rapid environmental scan methodology is that it relies heavily on web searches and databases, which are only as accurate and up to date as the information posted on websites and databases. Institutions may be slow to update their websites; therefore, some of the information found may be outdated and could bias the findings to only the most recent items 
[27]. Web-based searches are also limited in their ability to identify webinars and other similar training sessions, typically biasing the findings to only the most recent training sessions. Databases may not follow a standard data collection protocol; for example, when applying this method to the Canadian context we found that some CRCs may not have been captured in our search because the CRCs did not have any information beyond their name or chair title uploaded for our search string to pick up. Additionally, relying on the Global Health $\mathrm{MeSH}$ in the PubMed database may skew the assessment of research productivity in favour of certain kinds of research outputs. The MeSH captures "research on improving health and achieving equity in health for all people" but would not capture all of the biomedical, clinical, health systems and epidemiological research relevant to the field of global health. Developing a better, optimised PubMed search string for global health research that was both highly sensitive and specific would have been better but was beyond the scope of this rapid scan.

\section{Conclusions}

This study sought to develop a rapid environmental scan methodology to map where a country's global health research expertise lies, both geographically and substantively. Our framework and three-pronged approach was highly effective in responding to the real-time evidence needs of decision-makers in Canada. Other researchers can build on this approach to map expertise and research strengths in other settings and, where timelines allow, may consider adding more indicators (e.g. global health conferences/workshops as activities or registered patents as outputs) or incorporating additional data sources, including surveys of universities and keyinformant interviews with stakeholders.

\section{Supplementary information}

Supplementary information accompanies this paper at https://doi.org/10. 1186/s12961-020-0543-x.

Additional file 1. Workflow diagram of inputs, activities and outputs

\section{Abbreviations}

CIHR: Canadian Institutes of Health Research; CRC: Canada Research Chair; LMICs: Low- and middle-income countries

\footnotetext{
Acknowledgements

This research was supported by the International Collaboration for Capitalizing on Cost-Effective and Life-Saving Commodities (i4C) that is funded through the Research Council of Norway's Global Health \& Vaccination Programme (GLOBVAC Project \#234608). SJH is additionally funded by the Canadian Institutes of Health Research (CIHR) and the Ontario Government's Ministry of Research, Innovation \& Science.
}

\section{Authors' contributions}

SJH and SRVK made substantial contributions to the conception and design of the work. RN led the acquisition, analysis and interpretation of data. All authors drafted the work and substantively revised it. The author(s) read and approved the final manuscript.

\section{Funding}

This research funded through the Research Council of Norway's Global Health \& Vaccination Programme (GLOBVAC Project \#234608). SJH is additionally funded by the Canadian Institutes of Health Research (CIHR) and the Ontario Government's Ministry of Research, Innovation \& Science.

Availability of data and materials

All data generated or analysed during this study are included in this published article and its supplementary information files.

Ethics approval and consent to participate

Not applicable.

Consent for publication

Not applicable.

\section{Competing interests}

The authors declare that they have no competing interests.

\section{Author details}

${ }^{1}$ Global Strategy Lab, Dahdaleh Institute for Global Health Research, Faculty of Health, and Osgoode Hall Law School, York University, 4700 Keele Street 2120 Dahdaleh Building, Toronto, Ontario M3J 1P3, Canada. ${ }^{2}$ School of Epidemiology and Public Health, Faculty of Medicine, University of Ottawa, Ottawa, Ontario, Canada. ${ }^{3}$ Department of Global Health and Population, Harvard T.H. Chan School of Public Health, Harvard University, Boston, MA, United States of America. ${ }^{4}$ Department of Health Research Methods, Evidence and Impact and McMaster Health Forum, Faculty of Health Sciences, McMaster University, Hamilton, Ontario, Canada.

Received: 21 August 2019 Accepted: 21 February 2020

Published online: 09 April 2020

\section{References}

1. Viergever RF, Hendriks TCC. The 10 largest public and philanthropic funders of health research in the world: what they fund and how they distribute their funds. Health Res Policy Sys. 2016;14:12. https://doi.org/10.1186/ s12961-015-0074-Z.

2. Cartier Y, Creatore MI, Hoffman SJ, Potvin L. Priority-setting in public health research funding organisations: an exploratory qualitative study among five high-profile funders. Health Res Policy Syst. 2018;16:53. https://doi.org/10. 1186/s12961-018-0335-8.

3. M. Schäferhoff et al. Rethinking the Global Health System. 2015. https:// www.chathamhouse.org/publication/rethinking-global-health-system\# Accessed 4 Mar 2020.

4. Hoffman SJ. Strengthening global health diplomacy in Canada's foreign policy architecture: Literature review and key informant interviews. Can Foreign Policy J. 2010;16(3):17-41. https://doi.org/10.1080/11926422.2010. 9687318.

5. Plamondon K, Walters D, Campbell S, Hatfield J. Promoting equitable global health research: a policy analysis of the Canadian funding landscape. Health Res Policy Syst. 2017;15:72. https://doi.org/10.1186/s12961-017-0236-2.

6. Nixon SA, et al. Canada's global health role: supporting equity and global citizenship as a middle power. Lancet. 2018;391(10131):1736-48. https://doi. org/10.1016/S0140-6736(18)30322-2.

7. Hoffman SJ, Gunn E, Van Katwyk SR, Nixon SA. Systematic analysis of global health research funding in Canada, 2000-2016. Can J Public Health. 2020; 111:80-95.

8. International Development Research Centre. What we do. International Development Research Centre. https://www.idrc.ca/en/what-we-do. Accessed 16 Apr 2019.

9. Grand Challenges Canada. About Grand Challenges Canada. https://www. grandchallenges.ca. Accessed 16 Apr 2019.

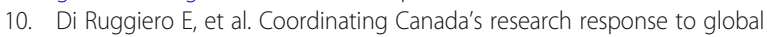
health challenges: the Global Health Research Initiative. Can J Public Health. 2006;97:29-31. 
11. Payne AA, Siow A. Does federal research funding increase university research output? Adv Econ Analysis Policy. 2003;3. https://doi.org/10.2202/ 1538-0637.1018.

12. Gonzalez-Brambila C, Veloso FM. The determinants of research output and impact: a study of Mexican researchers. Res Policy. 2007;36(7):1035-51. https://doi.org/10.1016/j.respol.2007.03.005.

13. Hirsch JE. An index to quantify an individual's scientific research output. Proc Natl Acad Sci USA. 2005;102(46):16569-72. https://doi.org/10.1073/ pnas.0507655102.

14. Government of Canada, Areas of Science, Canadian Institutes of Health Research. http://www.cihr-irsc.gc.ca/e/49834.html. Accessed 18 Jan 2018.

15. Universities Canada. Search Programs, UniversityStudy.ca. https://www. universitystudy.ca/search-programs/. Accessed 18 Jan 2018.

16. Government of Canada. Program Details, Canada Research Chairs. http:// www.chairs-chaires.gc.ca/program-programme/index-eng.aspx. Accessed 18 Jan 2018.

17. Government of Canada. About Us, Canada Research Chairs. http://www. chairs-chaires.gc.ca/about_us-a_notre_sujet/index-eng.aspx. Accessed 18 Jan 2018.

18. World Health Organization. World Health Organization Collaborating Centres. http://www.who.int/collaboratingcentres/en/. Accessed 18 Jan 2018.

19. World Health Organization, WHO Collaborating Centres Database. http:// www.who.int/collaboratingcentres/database/en/. Accessed 18 Jan 2018.

20. Brolan CE, et al. The right to health of non-nationals and displaced persons in the sustainable development goals era: challenges for equity in universal health care. Int J Equity Health. 2017;16:14. https://doi.org/10.1186/s12939016-0500-z.

21. Ruckert A, Labonte R. The financial crisis and global health: the International Monetary Fund's (IMF) policy response. Health Promot Int. 2013;28(3):35766. https://doi.org/10.1093/heapro/das016.

22. Kohler JC, Mackey TK, Ovtcharenko N. Why the MDGs need good governance in pharmaceutical systems to promote global health. BMC Public Health. 2014;14:63. https://doi.org/10.1186/1471-2458-14-63.

23. Molla YB, et al. Geographic information system for improving maternal and newborn health: recommendations for policy and programs. BMC Pregnancy Childbirth. 2017;17:26. https://doi.org/10.1186/s12884-016-1199-y.

24. Anderson I, et al. Indigenous and tribal peoples' health (The Lancet-Lowitja Institute Global Collaboration): a population study. Lancet. 2016;388:10040.

25. Walker IF, et al. Multidrug-resistant tuberculosis treatment programmes insufficiently consider comorbid mental disorders. Int J Tuberc Lung Dis. 2017;21:603-9. https://doi.org/10.5588/ijtld.17.0135.

26. Government of Canada. Marceline Côté, Canada Research Chairs. http:// www.chairs-chaires.gc.ca/chairholders-titulaires/profile-eng.aspx?profileld= 3420. Accessed 20 May 2019

27. Rogers Van Katwyk S, Jones SL, Hoffman SJ. Mapping educational opportunities for healthcare workers on antimicrobial resistance and stewardship around the world. Hum Resour Health. 2018;16:9. https://doi. org/10.1186/s12960-018-0270-3.

\section{Publisher's Note}

Springer Nature remains neutral with regard to jurisdictional claims in published maps and institutional affiliations.

Ready to submit your research? Choose BMC and benefit from:

- fast, convenient online submission

- thorough peer review by experienced researchers in your field

- rapid publication on acceptance

- support for research data, including large and complex data types

- gold Open Access which fosters wider collaboration and increased citations

- maximum visibility for your research: over $100 \mathrm{M}$ website views per year

At BMC, research is always in progress.

Learn more biomedcentral.com/submissions 